\title{
Eficiência da estimativa volumétrica para Goupia glabra em uma área de manejo florestal na Amazônia brasileira
}

\author{
Girlene da Silva Cruz ${ }^{*}$ João Ricardo Vasconcellos Gama ${ }^{2}$ Marcos Felipe Nicoletti ${ }^{1}$ Renato Bezerra da Silva \\ Ribeiro $^{2}$ Bruno Rafael Silva de Almeida ${ }^{1}$ Daniele Lima da Costa ${ }^{2}$ Lucas Cunha Ximenes ${ }^{2}$ Misael Freitas dos \\ Santos 3
}

${ }^{1}$ Universidade do Estado de Santa Catarina, Centro de Ciências Agroveterinárias, Av. Luiz de Camões, 2090 - Conta Dinheiro, Lages - SC, $88520-000$
${ }^{2}$ Universidade Federal do Oeste do Pará, Rua Vera Paz, s/n, Salé, Santarém-PA, 68040-255

${ }^{3}$ Universidade Estadual do Centro Oeste, campus Irati, PR 153, Km 7 s/n, Riozinho - PR, 84500-000

*Author for correspondence: scruz.girlene@ gmail.com

Received: February 2019 / Accepted: June 2019 / Published: June 2019

\section{Resumo}

Este estudo teve como objetivo avaliar a eficiência da estimativa volumétrica para Goupia glabra na Floresta Nacional do Tapajós. Foram utilizadas 227 árvores de Goupia glabra com $50 \mathrm{~cm} \geq \mathrm{DAP}<120 \mathrm{~cm}$, onde foram ajustados nove modelos matemáticos. Para a escolha do melhor modelo foram analisados a significância dos coeficientes pelo teste $\mathrm{t}$ a $95 \%$ de probabilidade, $\mathrm{R}^{2}$ ajustado\%, $\mathrm{S}_{\mathrm{y} . \mathrm{x} \%}$, análise gráfica e histogramas de resíduos. Foi realizado a validação da melhor equação através do teste Qui quadrado a $95 \%$ de probabilidade. Os modelos de Spurr variável combinada, Schumacher Hall e Spurr ambos logaritmizados, apresentaram os melhores resultados dos parâmetros de precisão. Ao analisar a distribuição gráfica e o histograma de resíduos, pode-se concluir que o modelo de Spurr variável combinada é o melhor para estimar o volume da espécie na área. $\mathrm{O}$ volume estimado pela equação não apresentou diferenças significativas quando comparados com o volume real, sendo validado a equação para a área de estudo.

Palavras-chave: Cupiúba, Flona Tapajós, modelos volumétricos.

\section{Abstract}

This study aimed to evaluate the efficiency of the volumetric estimation for Cupiúba in the Tapajos National Forest. We used 227 Goupia glabra trees with $50 \mathrm{~cm} \geq$ DAP $<120 \mathrm{~cm}$, where nine mathematical models were fitted. For the choice of the best model, the significance of the coefficients was analyzed by $t$ test at $95 \%$ probability, $\mathrm{R}^{2}$ adjusted\%, $\mathrm{S}_{\mathrm{y} . \mathrm{x} \%}$, graphical analysis and histograms of residues. The best equation was validated through the chisquare test at $95 \%$ probability. The combined Spurr variable models, Schumacher Hall and Spurr both logarithmized, presented the best results of the precision parameters. When analyzing the graphical distribution and the histogram of residues, it can be concluded that the combined variable Spurr model is the best to estimate the volume of the species in the area. The volume estimated by the equation did not present significant differences when compared with the real volume, and the equation for the study area was validated.

Key words: Cupiúba, Flona Tapajos, volumetric models.

\section{Introdução}

O manejo de florestas naturais e sua conservação, demandam conhecimentos sobre a sua estrutura e estoques correntes, bem como sobre os fatores que determinam a sua evolução ao longo dos anos. Para se obter informações referente a volumetria da floresta é necessário a execução de inventários florestais, onde, com base em variáveis coletadas, são ajustados modelos volumétricos para estimar a volumetria da área que será manejada (Soares et al. 2011; Silva e Santana 2014).

A correta estimativa de volumes comerciais ou totais faz com que as empresas tomem decisões mais precisas referente as análises de investimentos e volumetria a ser retirada a cada ano (Rufini et al. 2010). Além disto, a estimativa correta do volume comercial determina as dimensões da madeira colhida e têm grande influência no rendimento econômico da empresa (Stepka et al. 2017).

A estimativa de volume total ou comercial são variáveis de grande interesse a serem estimadas em florestas sujeitas à prática do manejo florestal sustentável. Estimando-se estes volumes com precisão, os planos de manejo, as análises de investimentos e as tomadas de decisão também serão precisos (Rufini et al. 2010).

Para estimativa do volume de árvores, é utilizado equações em que o volume constitui a variável dependente, estando as variáveis independentes comumente representadas pelo diâmetro à altura do peito e à altura total (Machado et al. 2002). Mas, devido à altura ser uma variável de difícil obtenção em florestas naturais densas e de dossel superior elevado (Soares et al. 2011) passou a se utilizar dados de romaneio para o ajuste de equações volumétricas, onde, os comprimentos das toras correspondem a altura comercial dos indivíduos (SilvaRibeiro et al. 2014).

Para Cysneiros et al. (2017) no contexto das concessões florestais, o desenvolvimento de equações de volume para espécies florestais amazônicas, é necessário para subsidiar as ações de planejamento e ordenamento florestal. Diversas etapas do manejo em regime de concessão requerem estimativas precisas do volume comercial das árvores, desde a avaliação da floresta para o embasamento das licitações, até a emissão da Autorização de Exploração por parte dos órgãos ambientais competentes, justificando assim o aprimoramento dessas equações.

Na região oeste do Pará, a Cooperativa Mista da Flona Tapajós (Coomflona) maneja anualmente uma área de 1.600 ha e utiliza uma equação desenvolvida especificamente para a área a ser manejada. Anualmente esta equação é reajustada visando melhorar a estimativa do volume dessas espécies. Alguns autores demostraram que o ajuste de equações volumétricas, desenvolvidas especificamente para as áreas de manejo florestal, constitui o procedimento mais eficiente, econômico e com precisão aceitável para a quantificação da produção madeireira da floresta (Barros e Silva-Junior 2009). Porém, equações de volume tendem a ter suas aplicações restritas a uma determinada área, idade, espaçamento, rotação ou espécie 
(Couto e Bastos 1987). Isto ocorre devido as diferentes condições de clima e solo influenciarem no crescimento e relação entre as variáveis da árvore.

Dessa forma, a criação de modelos baseados em dados de um povoamento ou região, sujeita a condições uniformes de clima e de solo, permite aos produtores florestais ferramentas que possibilitam a estimação mais exata das variáveis nesse povoamento ou região (Valente et al. 2011; Soares et al. 2011). Diante disto, o objetivo do presente estudo foi avaliar a eficiência da estimativa volumétrica para Goupia glabra na Floresta Nacional do Tapajós.

\section{Material e métodos \\ Área de estudo}

O estudo foi realizado na Floresta Nacional do Tapajós (Flona Tapajós), especificamente na Área de Manejo Florestal da Cooperativa Mista da Flona do Tapajós, conforme figura 1 .

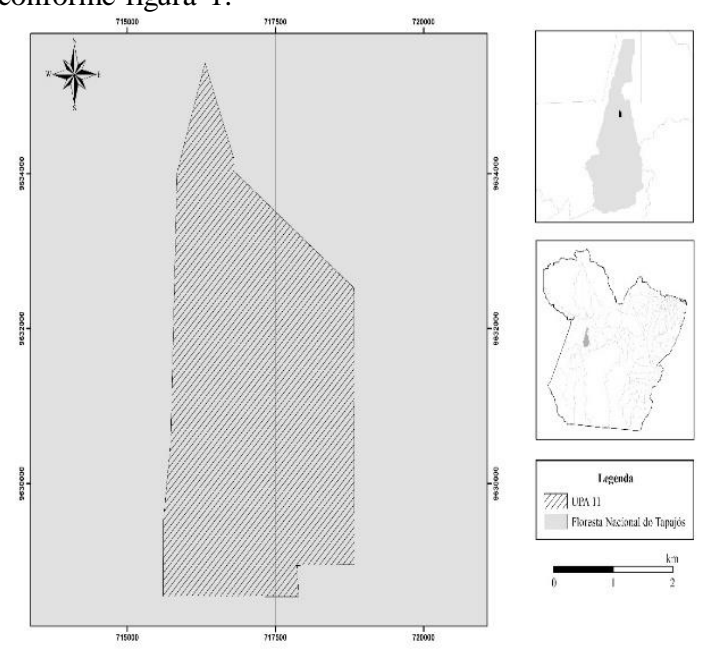

Figura 1. Mapa de localização da Unidade de Produção Anual 11, na Floresta Nacional do Tapajós, km 117.

O clima da região é classificação por Köppen do tipo $\mathrm{Am}$, com índice pluviométrico superior a $2.300 \mathrm{~mm}$ por ano e temperatura média de 22 e $25^{\circ} \mathrm{C}$ (Alvares et al. 2013). Nos meses de dezembro a maio há uma maior ocorrência de chuvas e de julho a agosto uma queda pluviométrica brusca, caracterizando-se um período seco onde a precipitação mensal é inferior a $60 \mathrm{~mm}$ (Inmet 2015). A vegetação predominante é a de Floresta Ombrófila Densa, com árvores de grande porte variando de 25 a $40 \mathrm{~m}$ de altura (Pinho et al. 2009). Nessa região, há predominância de Latossolo Amarelo Distrófico, caracterizados por diferentes texturas, geralmente profundo, ácido, friável e revestido por florestas densas (Espírito-Santo et al. 2005).

\section{Coleta de dados}

Foram utilizadas nos ajustes 227 árvores que romaneadas de Goupia glabra com $50 \mathrm{~cm} \geq \mathrm{DAP}<120$ $\mathrm{cm}$, cubadas pelo método de Smalian e conforme metodologia adotada por Silva-Ribeiro et al. (2014), a soma dos comprimentos das toras, corresponderam à altura comercial de cada árvore. Durante o romaneio realizado pela Coomflona as toras são divididas em seções que variam de 4 a $7 \mathrm{~m}$, para melhorar o aproveitamento do fuste e também atender as demandas do transporte durante o baldeio (Gomes et al. 2018). Com os dados observados de volume real, foram ajustados 8 modelos volumétricos para a espécie (Tabela 1).
Tabela 1. Modelos volumétricos testados para Goupia glabra, em uma unidade de manejo florestal, na Floresta Nacional do Tapajós.

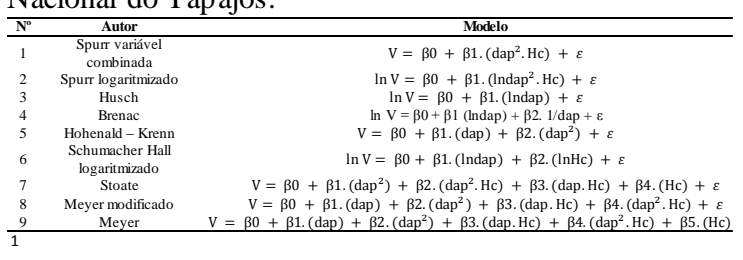

Em que: $V=$ volume com casca, em $\mathrm{m}^{3}$; dap= diâmetro a $1,30 \mathrm{~m}$ do solo, em $\mathrm{cm} ; \mathrm{Hc}=$ soma do comprimento das toras, em $\mathrm{m} ; \beta 0$, $\beta 1, \beta 2, \beta 3, \beta 4, \beta 5=$ coeficientes da regressão; ln = logaritmo neperiano; $\varepsilon=$ erro aleatório.

A escolha do melhor modelo foi baseada nos parâmetros de precisão: coeficiente de determinação ajustado ( $\mathrm{R}^{2}$ ajustado\%), erro padrão da estimativa $\left(\mathrm{S}_{\mathrm{y} . \mathrm{x} \%}\right)$, análise gráfica dos dados ajustados e dos resíduos e histograma de resíduos.

A multicolinearidade foi analisada por meio do VIF (Variance inflation factor) para modelos que apresentam mais de uma variável independente. Geralmente, VIF > 10 significa que o modelo apresenta multicolinearidade problemática, ou seja, a correlação entre as variáveis está afetando os valores dos coeficientes dos modelos. Segundo Draper e Smith (1980) valores de VIF menores que 1 demonstram que não existe multicolinearidade; valores entre 1 e 10 demonstram que existe multicolinearidade aceitável e; valores de VIF acima de 10, demonstram uma multicolinearidade problemática.

Para a comparação entre as equações logarítmicas e não logarítmicas, o erro padrão e o coeficiente de determinação ajustado foram recalculados. Os modelos volumétricos foram ajustados pelo método dos mínimos quadrados ordinários (MMQO).

O processamento, análise de dados e os testes aplicados foram realizados utilizando planilha eletrônica do Microsoft Excel 2013 e a extensão Action 2.8.

\section{Validação da melhor equação}

Realizou-se a validação da melhor equação com 50 árvores, que não fizeram parte dos ajustes. $\mathrm{O}$ volume real e o estimado foram comparados pelo teste Qui quadrado a $95 \%$ de probabilidade.

\section{Resultados e Discussão} Estatística descritiva

O volume total mensurado para a espécie foi de $997,3004 \mathrm{~m}^{3}$, com um erro padrão de $1,94 \mathrm{~m}^{3}$, sendo o menor volume observado de $1,7164 \mathrm{~m}^{3}$ e o maior de $12,9994 \mathrm{~m}^{3}$. A média dos diâmetros dos indivíduos foi de $72,11 \mathrm{~cm}$, sendo que o menor diâmetro encontrado foi de $50,93 \mathrm{~cm}$ e maior foi de $120,96 \mathrm{~cm}$. Em relação à altura comercial desses indivíduos, a média foi de $15,65 \mathrm{~m}$ e variaram entre $6,10 \mathrm{~m}$ à $23,65 \mathrm{~m}$.

Na tabela 2 se encontram a estatística descritiva dos indivíduos cubados. Nota-se uma alta correlação entre as variáveis diâmetro e volume, porém, é notável a baixa correlação da variável diâmetro e altura.

Tabela 2. Estatística descritiva da população amostrada de Goupia glabra, em uma unidade de manejo florestal, naFloresta Nacional do Tapajós. 


\begin{tabular}{|c|c|c|c|c|c|}
\hline & \multirow{2}{*}{$\bar{Y}$} & \multirow{2}{*}{ s } & \multirow{2}{*}{$\mathrm{s}^{2}$} & \multicolumn{2}{|r|}{$\mathrm{r}$} \\
\hline & & & & $d x v$ & $\mathrm{dxhc}$ \\
\hline Cupiúba & 4,8413 & 1,9470 & 3,7909 & 0,7183 & 0,1536 \\
\hline
\end{tabular}

Em que: $\overline{\mathrm{Y}}=$ média dos volumes reais em $\mathrm{m}^{3} ; \mathrm{s}=$ desvio padrão; $\mathrm{s}^{2}=$ variância; $\mathrm{r}=$ coeficiente de correlação linear de Pearson; $\mathrm{d}=$ diâmetro à altura do peito; hc = altura comercial; $\mathrm{e} \mathrm{v}=$ volume real.

\section{Equações de volume para a espécie}

Após os ajustes dos modelos volumétricos, foi possível observar que os modelos de dupla entrada apresentaram os melhores parâmetros de precisão quando comparados com os de simples entrada, conforme demonstrado na tabela 3. Em relação a significância dos coeficientes pelo teste " $t$ " é nítido que modelos tidos como mais simples, apresentaram todos seus coeficientes significativos. A não significância de coeficientes das equações pode ser indício de multicolinearidade problemática nos dados utilizados.

Tabela 3. Parâmetros dos ajustes e estatísticas de precisão para estimativas volumétricas de Goupia glabra, em uma unidade de manejo florestal, na Floresta Nacional do Tapajós.

\begin{tabular}{|c|c|c|c|c|}
\hline $\begin{array}{l}\text { Modelo } \\
\end{array}$ & & icientes & $\frac{R^{2}{ }_{\text {ajustado } \%}}{4}$ & $\mathbf{S}_{y \cdot x_{\%}}$ \\
\hline Spurr variável combinada & $\beta_{\beta 1}^{\beta 0}$ & $0,76077^{*}$ & 89,60 & 17,54 \\
\hline Spurr logaritmizado & $\begin{array}{l}\beta 0 \\
\beta 1\end{array}$ & $\begin{array}{l}-7,4871^{*} \\
0,7982^{*}\end{array}$ & 88,86 & 17,89 \\
\hline Husch & $\begin{array}{l}\beta 0 \\
\beta 1\end{array}$ & $\begin{array}{l}-5,9732^{*} \\
1,7529^{*}\end{array}$ & 61,58 & 28,34 \\
\hline Brenac & $\begin{array}{l}\beta 0 \\
\beta 1 \\
\beta 2\end{array}$ & $\begin{array}{l}-7,4269^{\text {ns }} \\
2,0275^{\mathrm{ns}} \\
19,874^{\mathrm{nn}}\end{array}$ & 51,35 & 28,46 \\
\hline Hohenald - Krenn & $\begin{array}{l}\beta 0 \\
\beta 1 \\
\beta 2\end{array}$ & $\begin{array}{l}-3,3448^{\text {ns }} \\
0,1089^{\text {s }} \\
0,0000^{\text {sn }}\end{array}$ & 51,13 & 28,12 \\
\hline Schumacher Hall logaritmizado & $\begin{array}{l}\beta 0 \\
\beta 1 \\
\beta 2\end{array}$ & $\begin{array}{c}-7,4664^{*} \\
1,5797 * \\
0,8166^{*}\end{array}$ & 89,02 & 17,95 \\
\hline Stoate & $\begin{array}{l}\beta 0 \\
\beta 1 \\
\beta 2 \\
\beta 3 \\
\beta 4\end{array}$ & 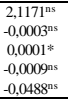 & 68,35 & 22,63 \\
\hline Meyer modificado & $\begin{array}{l}\beta \\
\beta 1 \\
\beta 2 \\
\beta 3 \\
\beta 4 \\
\end{array}$ & 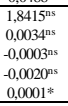 & 68,33 & 22,63 \\
\hline Meyer & $\begin{array}{l}\beta 0 \\
\beta 1 \\
\beta 2 \\
\beta 3 \\
\beta 4 \\
\beta 5\end{array}$ & 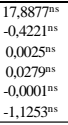 & 68,42 & 22,60 \\
\hline
\end{tabular}

Em que: $\mathrm{NA}=$ não se aplica; $\beta 0, \beta 1, \beta 2, \beta 3, \beta 4, \beta 5=$ coeficientes da regressão; $\mathrm{ns}=$ coeficientenão significativo, de acordo com o teste $\mathrm{t}$ a $95 \%$ de probabilidade; $*=$ coeficiente significativo, de acordo com o teste $\mathrm{t}$ a $95 \%$ de probabilidade; $\mathrm{S}_{\mathrm{y} . \mathrm{x} \%}=$ erro padrão da estimativa.

Apenas o modelo de Schumacher Hall apresentou multicolinearidade inferior a 10 para ambas variáveis independentes, conforme apresentado na tabela 4. Os modelos que apresentaram coeficientes não significativo s apresentaram elevados valores de multicolinearidade para suas variáveis independentes.

T abela 4. Tabela de análise de multicolinearidade para os modelos com duas ou mais variáveis independentes testados para Goupia glabra, em unidade de manejo florestal, na Floresta Nacional do Tapajós.

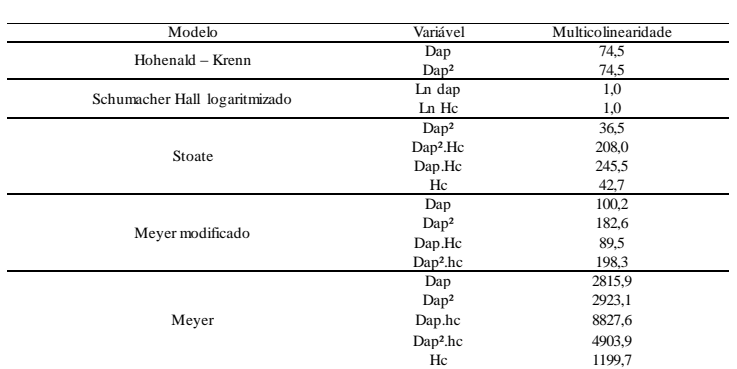

A análise gráfica de resíduos (figura 2) demonstra se as distribuições obtiveram alguma tendenciosidade, sendo considerada importante na tomada de decisões para a escolha do melhor modelo, pois através dessa análise será possível afirmar se os modelos subestimaram ou superestimaram as estimavas volumétricas para a espécie na área de estudo.

Os modelos de Spurr varável combinada e Spurr e Schumacher ambos logaritmizados apresentaram as melhores distribuições de resíduos, estando a dispersão dos dados concentrados mais próximos a linha regressora, demonstrando que o modelo não apresentou nenhuma tendência de sub ou superestimativa. Quando analisado o histograma de resíduos, é possível identificar que o modelo de Spurr logaritmizado tende a subestimar os volumes de alguns indivíduos, já Spurr variável combinada e Schumacher Hall apresentam maior concentração de indivíduos na classe de erro percentual zero.

A distribuição do volume estimado em relação ao volume observado demonstra tendências semelhantes para os três modelos, onde os valores estimados estão bem próximos dos valores reais obtidos durante a cubagem. 
$1-\mathrm{A}$

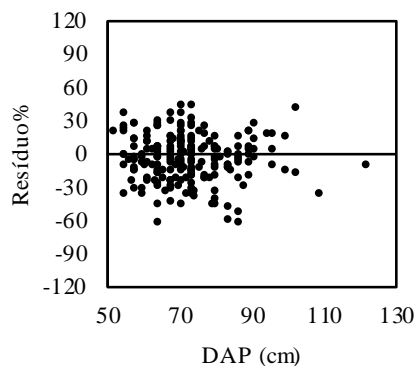

1 - B

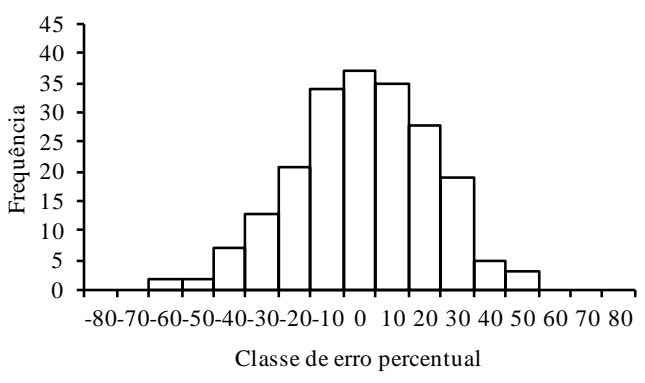

1 - C

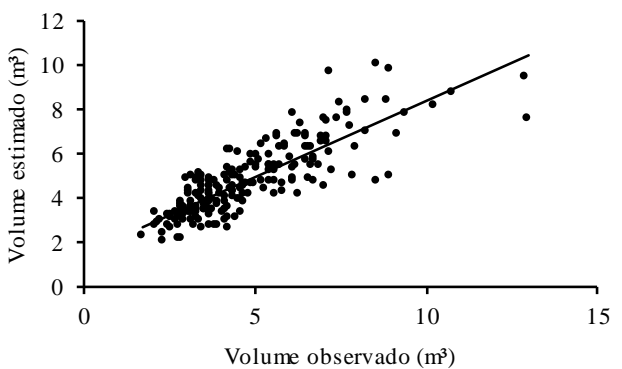

$2-A$

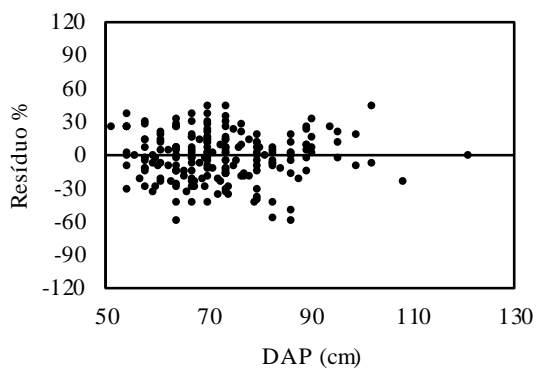

2 - B

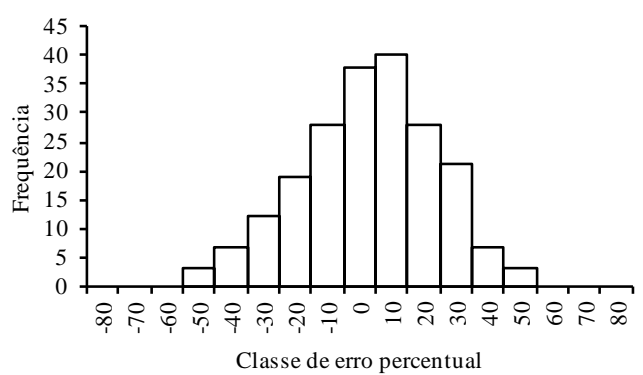

$2-C$

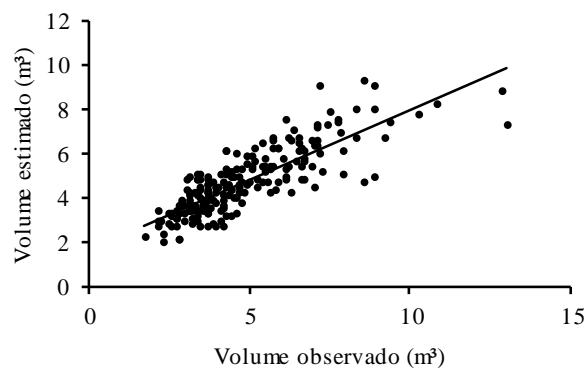


$3-\mathrm{A}$

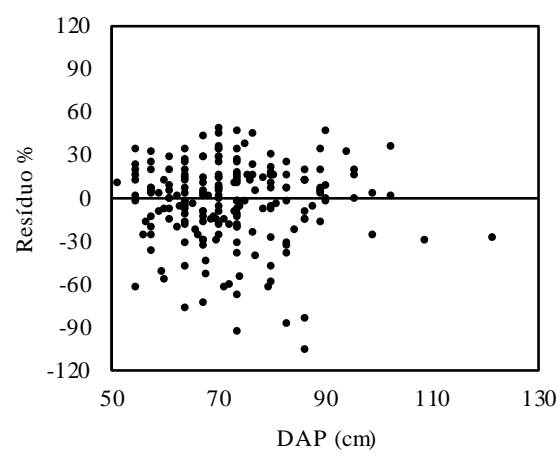

$3-B$

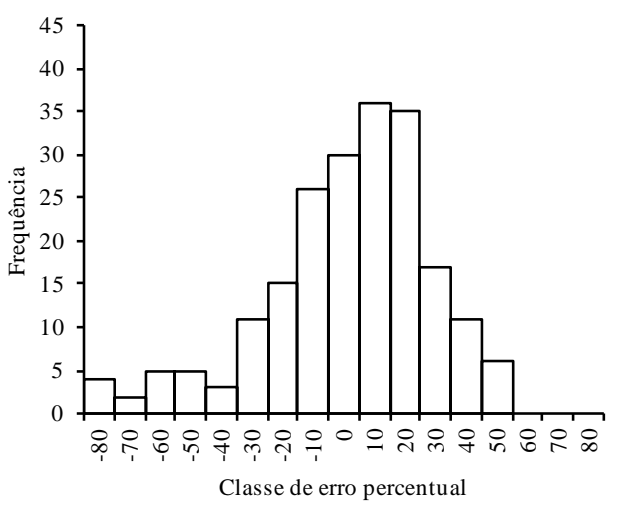

$3-\mathrm{C}$

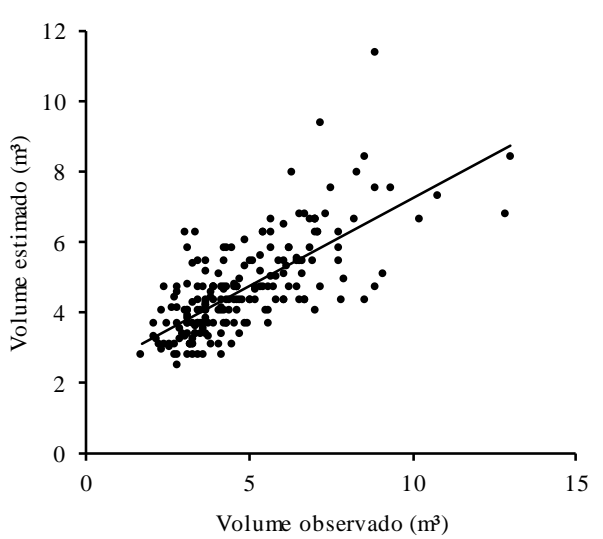

4 - A

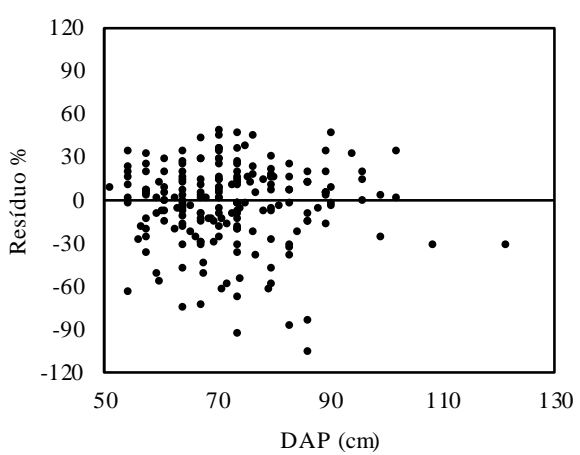

4 - B

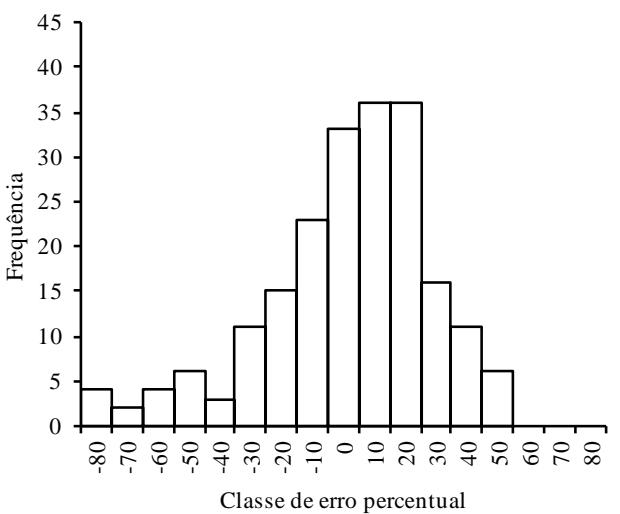

4 - C

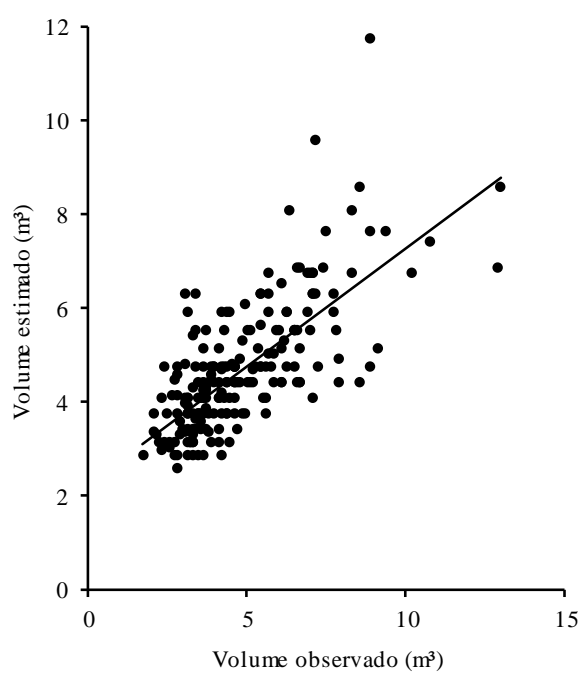


$5-\mathrm{A}$

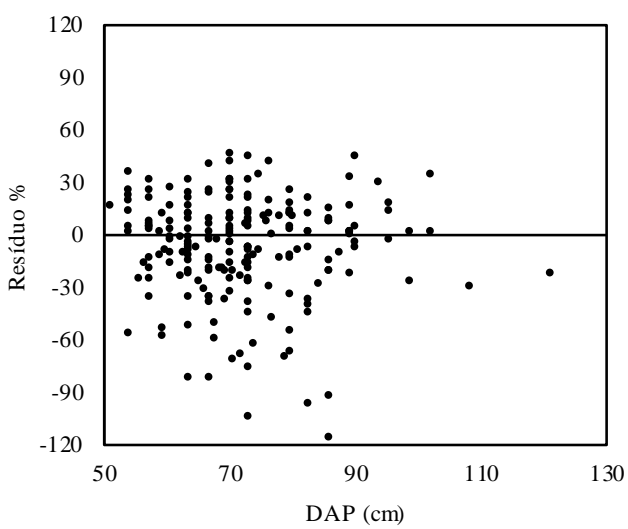

5 - B

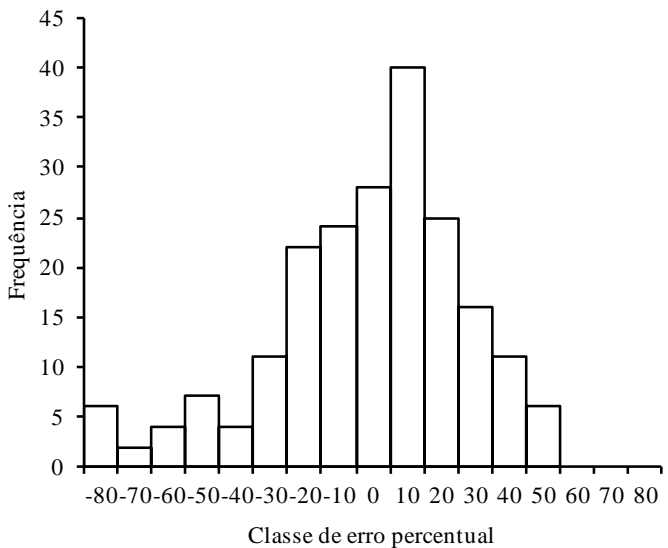

5 - C

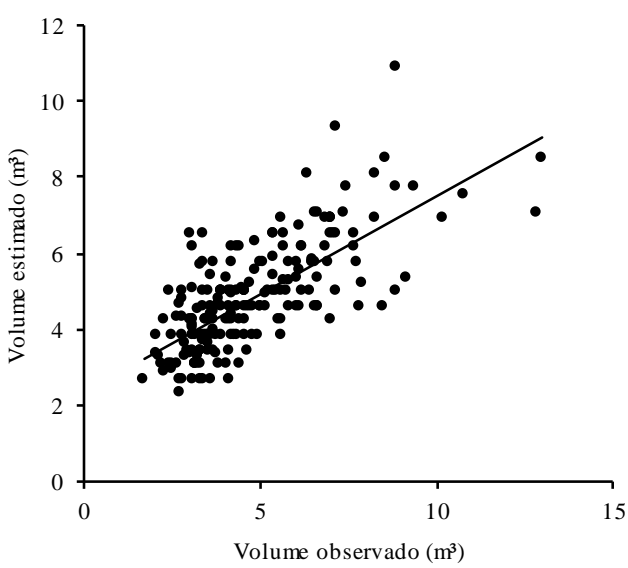

6-A

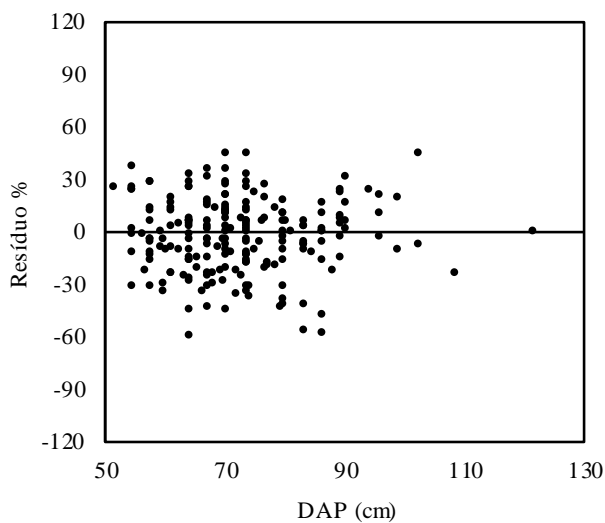

6 - B

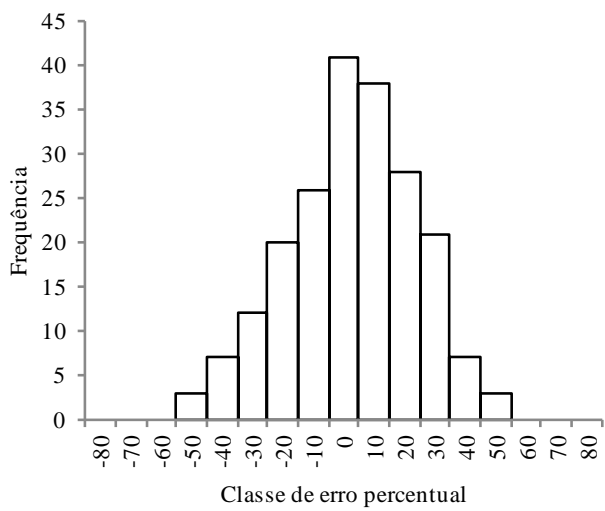

6-C

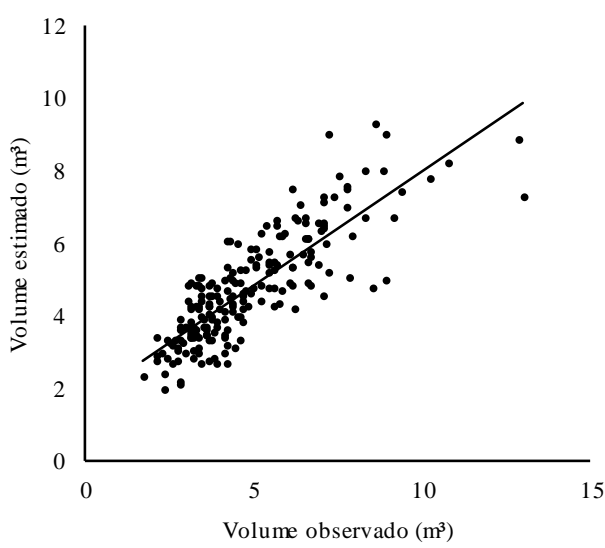




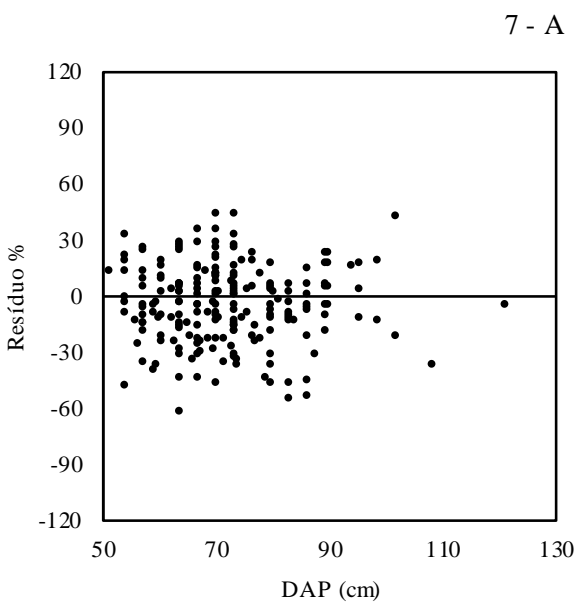

7 - B

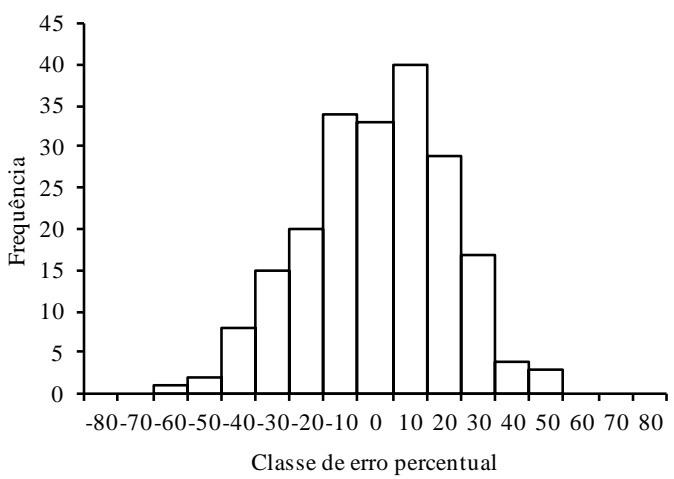

$7-\mathrm{C}$

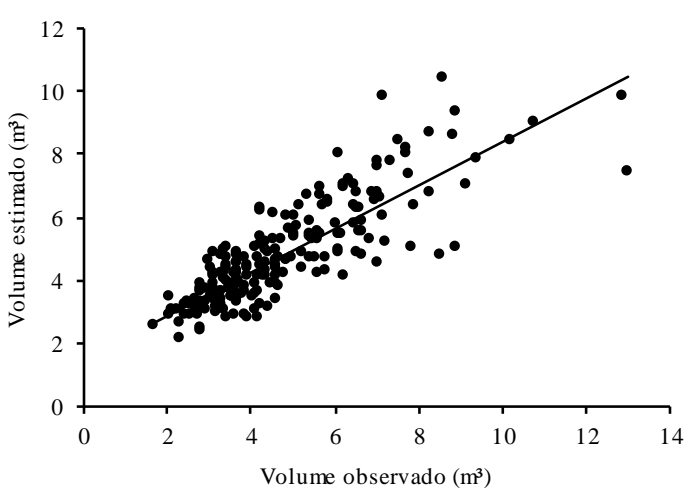

$8-\mathrm{A}$

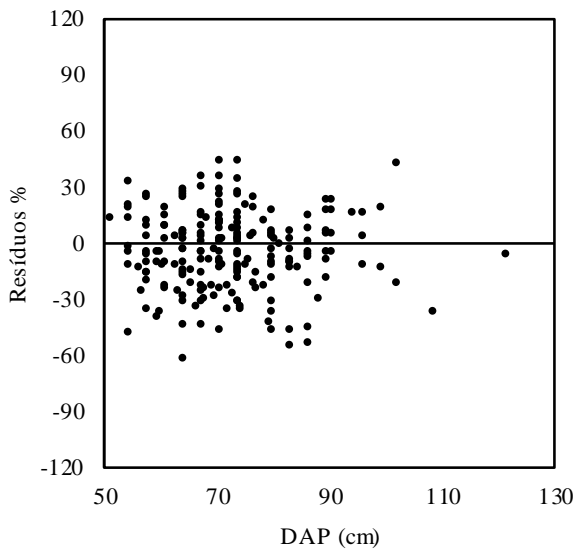

8 - B

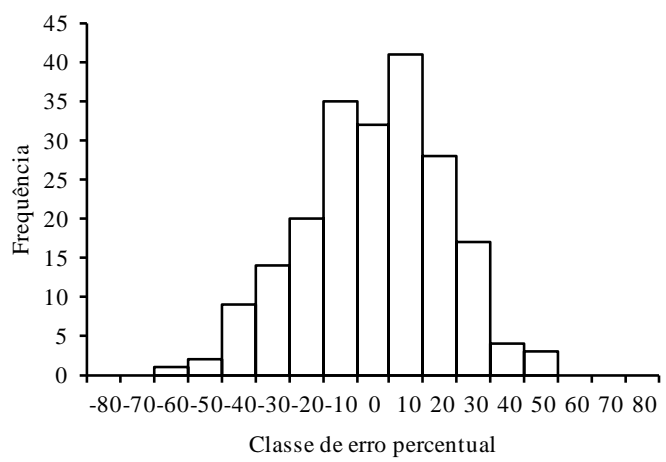

$8-\mathrm{C}$

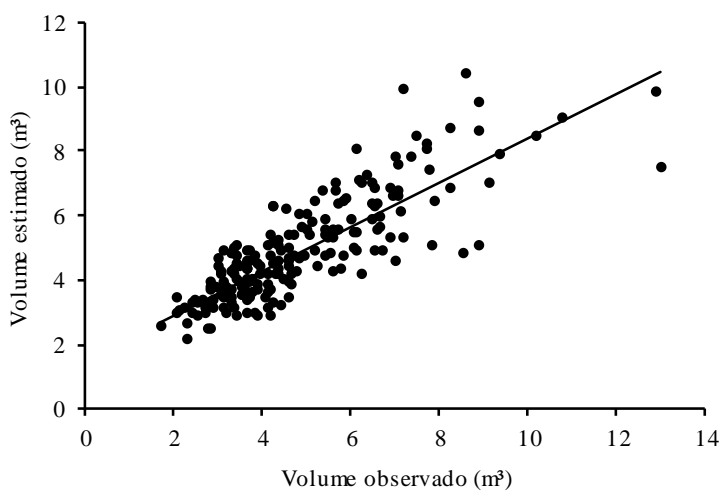




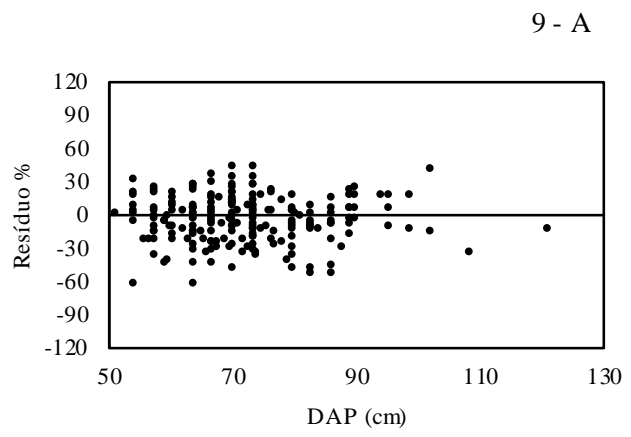

$9-\mathrm{B}$

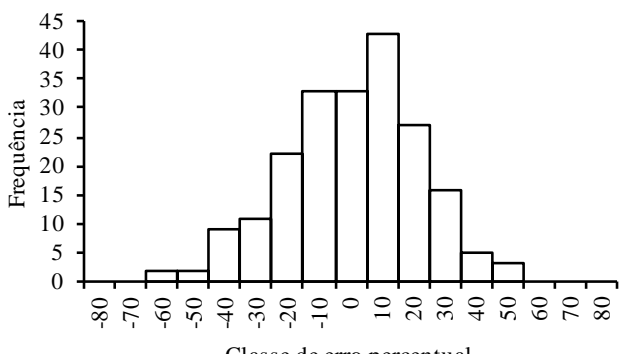

$9-\mathrm{C}$

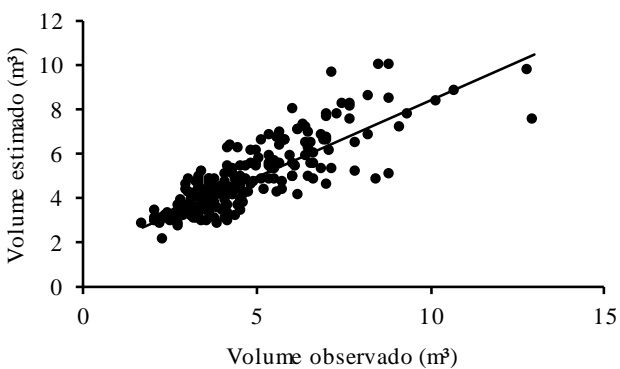

Figura 2. Distribuição gráfica para os modelos ajustados para Goupia glabra, em uma unidade de manejo florestal, na Floresta Nacional do Tapajós.

Em que: A - Distribuição gráfica dos resíduos; B - histograma de resíduos; $\mathrm{C}$ - volume observado $\mathrm{x}$ volume estimado. 1 - Spurr variável combinada; 2 - Spurr logaritmizado; 3 - Husch; 4 Brenac; 5 - Hohenald - Krenn; 6 - Schumacher Hall logaritmizado; 7 - Stoate; 8 - Meyer modificado; 9 - Meyer.

\section{Validação da melhor equação}

Ao comparar os volumes reais e estimados pela equação de Spurr variável combinada pode-se concluir que não existem diferenças significativas entre os volumes, com p-valor de 0,6208 , a um de $95 \%$ de probabilidade. A equação $\mathrm{v}=0,76072+0,00004 *$ dap $^{2 *}$ hc pode ser utilizada para estimar a volumetria de Goupia glabra na área de manejo florestal, da Flona Tapajós.

\section{Discussão}

\section{Equações de volume para a espécie}

Cysneiros et al. (2017) afirma que é necessário o desenvolvimento de equações de volume para espécies e formações florestais amazônicas. Equações gerais ou genéricas, em muitos casos não estimam de forma satisfatória a volumetria de todas as espécies exploradas na Amazônia devido as características específicas das espécies. Alguns autores demonstram a necessidade de se estudar espécies individualmente ou até mesmo grupos de espécies com características semelhantes (Rolim et al. 2006; Soares et al. 2011; Silva Ribeiro et al. 2014).

Em relação a significância dos parâmetros pelo testet, Batista et al. (2004) encontraram resultados semelhantes ao do presente estudo, onde os modelos de Meyer e Stoate, modelos com muitas variáveis correlacionadas tiveram sérios problemas com a significância dos seus coeficientes, sendo atribuído esse resultado a possível multicolinearidade problemática existente nos dados.

A multicolinearidade é um problema que afeta significativamente os coeficientes da regressão, quando ocorre alta correlação entre as variáveis independentes, os coeficientes de regressão estimados tendem a ser imprecisos e as estimativas dos coeficientes podem oscilar consideravelmente de um subconjunto de dados para outro (Gonzáles e Formoso 2000). Ferreira (2009) aconselha que em casos de identificação de multicolinearidade problemática não seja utilizado o modelo, pois não se pode confiar fielmente nos valores dos coeficientes encontrados pela regressão.

Os modelos de dupla entrada apresentaram os melhores resultados em relação aos parâmetros de precisão. Thaines et al. (2010) identificaram os modelos de Husch e Schumacher e Hall como os melhores modelos para estimar o volume comercial de espécies comerciais no município de Lábrea, Amazonas. Silva Ribeiro et al. (2014) ao ajustar modelos matemáticos para 3 espécies nativas da Amazônia, identificaram que os modelos de Schumacher Hall e Spurr logaritmizados como os melhores para estimar a volumetria das espécies Maçaranduba, Jarana e Jatobá na Floresta Nacional do Tapajós. Tonini e Borges (2005) ajustando modelos matemáticos para estimar o volume de espécies em Roraima observaram a superioridade de modelos de dupla entrada que utilizaram a variável altura comercial.

A superioridade dos modelos de dupla entrada em relação aos modelos de simples está relacionada à inserção da variável altura total ou comercial (Cysneiros et al. 2017). Modelos que apresentam mais de uma variável independente é considerado mais eficiente, uma vez que esses modelos resultam em estimativas mais precisas e não tendenciosas, uma vez que o volume é melhor explicado em relação das variáveis diâmetro e altura seja ela comercial ou total (Silva et al. 1984; Guimarães e Leite 1996; Campos e Leite 2009).

A inserção da variável altura melhora significativamente os resultados dos parâmetros de precisão dos modelos, mas vale se tomar cuidado com a utilização dessa variável, uma vez que em campo, a equipe de inventário é induzida a superestimar a altura comercial das espécies. Essa superestimação pode representar um aumento significativo nas estimativas de volume (Gimenez et al. 2015). Para contornar o problema da superestimativa das alturas em inventários na Amazônia, Silva Ribeiro et al. (2014) propuseram a utilização da soma do comprimento das toras do romaneio como altura comercial, metodologia adotada neste estudo.

A análise de resíduos junto com os parâmetros de precisão fornece importantes requisitos para a escolha do melhor modelo, pois através da distribuição gráfica é possível assegurar se as equações apresentam algum tipo de tendenciosidade (Miranda et al 2014; Cysneiros et al. 2017). 
Validação da melhor equação

A escolha de modelos mais simples, como o de Spurr com apenas uma variável combinada (altura e diâmetro à altura do peito), necessitam de um menor esforço computacional, são mais gerais e não sofrem com o problema de instabilidade dos parâmetros estimados, ou seja, a multicolinearidade (Couto e Bastos 1987). O modelo de Spurr a variável independente oferece maior peso ao diâmetro, elevando-o ao quadrado. Como a variável diâmetro apresenta elevada correlação com o volume, esse modelo apresenta vantagem, corrigindo parte dos problemas de superestimativa (Cy sneiros 2016).

\section{Conclusão}

O modelo de Spurr logaritmizado se apresentou como o melhor para a estimar o volume da espécie na Floresta Nacional do Tapajós;

A equação $\operatorname{lnv}=-7,48711284+0,798172345^{*}$ lndap ${ }^{2 *}$ hc é válida para estimar a volumetria da espécie.

\section{Referências}

Alvares CA, Stape JL, Sentelhas PC, Gonçalves JLM, Sparovek G (2013) Koppen's climate classification map for Brazil. Meteorologische Zeitschrift, 22(6): 711-728.

Barros PLC, Silva-Júnior AT (2009) Equação de volume para árvores de uma floresta tropical densa no município de Anapu, oeste do estado do Pará, Amazônia oriental. Revista de Ciências Agrárias, 51: 115-126.

Batista JLF, Marquesini M, Viana VM (2004) Equações de volume para árvores de caxeta (Tabebuia cassinoides) no Estado de São Paulo e sul do Estado do Rio de Janeiro. Scientia Forestalis, 65: 162-175.

Campos JCC, Leite HG (2009) Mensuração florestal: perguntas e respostas. 3 ed. Viçosa: Editora UFV, 542 p.

Couto HTZ, Bastos NLM (1987) Modelos de equações de volume e relação hipsométricas para plantações de Eucalyptus no Estado de São Paulo. IPEF, 37: 33-44.

Cysneiros VC (2016) Estratégias para modelagem do volume comercial em florestas tropicais. Dissertação. Universidade Federal do Paraná, Curitiba, 117p.

Cysneiros VC, Pelissari AL, Machado AS, Figueiredo Filho A, Souza L (2017) Modelos genéricos e específicos para estimativa do volume comercial em uma floresta sob concessão na Amazônia. Scientia Forestalis, 45: (114) 19.

Draper NR, Smith H (1980) Applied regression analisys. 2. Ed. New York: J. Wiley, 790p.

Espirito-Santo FDB, Shimabukuro YE, Oliveira LE, Aragão C, Machado ELM (2005) Análise da composição florística e fitossociológica da floresta nacional do Tapajós com o apoio geográfico de imagens de satélites. Acta Amazonica, 35: 155-173

Ferreira MZ (2009) Modelagem da influência de variáveis ambientais no crescimento e na produção de Eucalyptus sp. Tese. Universidade Federal de Lavras, Lavras, 112p.

Gimenez BO, Danielli FE, Oliveira CKA, Santos JS, Higuchi N (2015) Equações volumétricas para espécies comerciais madeireiras do sul do estado de Roraima. Scientia Forestalis, Piracicaba, 43(106): 291-301.
Gomes KMA, Silva Ribeiro RB, Gama JRV, Andrade DFC (2018) Eficiência na estimativa volumétrica de madeira na Floresta Nacional do Tapajós. Nativa 6(2): 170-176.

González MAS, Formoso CT (2000) Análise conceitual das dificuldades na determinação de modelos de formação de preços através de análise de regressão. Revista Engenharia Civil, 8: 95-75.

Guimarães DP, Leite HG (1996) Influência do número de árvores na determinação de equação volumétrica para Eucalyptus grandis. Scientia Forestalis, 50: 37-42.

$\begin{array}{lrrr}\text { Instituto Nacional dee } & \text { Meteorologia. } & \text { Normais } \\ \text { climatológicas, } & 2012 . & \text { Disponível } & \text { em: }\end{array}$ <www.inmet.gov.br>. Acesso em 23 jul. 2017.

Machado AS, Téo SJ, Urbano E, Figura MA, Silva LCR (2006) Comparação de métodos de cubagem absolutos com o volume obtido pelo xilômetro para Bracatinga (Mimosa scabrella Bentham). Revista Cerne, 12: (12) 239253.

Miranda LC, Paro BAV, Costa GR (2014) Estimativa do volume em árvores de Hymenaea coubaril L. e Trattinnickia burserifolia Mart. no norte de Mato Grosso. Nativa, 2(4):219-223.

Pinho GSC, Fiedler NC, Guimarães PP, Silva GF, Santos J (2009) Análise de custos e rendimentos de diferentes métodos de corte de cipós para produção de madeira na floresta nacional do Tapajós. Acta Amazonica, 39(3): 555560.

Rolim SG, Couto HTZ, Jesus RM, França JT (2006) Modelos volumétricos para a Floresta Nacional do Tapirapé-Aquirí, Serra dos Carajás (PA). Acta Amazonica, 36(1):107-114.

Rufini AL, Scolforo JRS, Oliveira AD, Mello JM (2010) Equações volumétricas para o cerrado Sensu stricto, em Minas Gerais. Cerne, Lavras, 16: (1) 1-11.

Silva EM, Santana AC (2014) Modelos de regressão para estimação do volume de árvores comerciais, em florestas de Paragominas. Revista Ceres, 61(5): 631-636.

Silva JNM, Carvalho JOP, Lopes JCA, Carvalho MSP (1984) Equações de volume para a floresta nacional do Tapajós. Boletim de Pesquisa Florestal, 8: 50-63.

Silva Ribeiro RB, Gama JRV, Melo LO (2014) Seccionamento para cubagem e escolha de equações de volume para a floresta Nacional do Tapajós. Cerne, 20(4): $605-612$.

Soares CPB, Martins FB, Leite Junior HU, Silva GF, Figueiredo LTM (2011) Equações hipsométricas, volumétricas e de Taper para onze espécies nativas. Revista Árvore, 35(5): 1039-1051.

Stepka TF, Zeny Junior GA, Lisboa GS, Cerqueira CL, Pesck VA, Roik M (2017) Modelos volumétricos e funções de afilamento para Pinus taeda L. na região dos Campos Gerais, Paraná, Brasil. Revista Spacios, 38(21): 19.

Thaines F, Braz EM, Mattos PP, Thaines AAR (2010) Equações para estimativa de volume de madeira para a região da bacia do Rio Ituxi, Lábrea, AM. Pesquisa Florestal Brasileira, Colombo, 30: 283-289. 
Tonini H, Borges RA (2015) Equação de volume para espécies comerciais em Floresta Ombrófila Densa no Sul de Roraima. Pesquisa Florestal Brasileira, 35: 111-117.

Valente DR, Queiroz WT, Pinheiro JG, Monteiro LAS (2011) Modelo de predição para o volume total de Quaruba (Vochysia imundata ducke) via análise de fatores e regressão. Revista Árvore, 35(2): 307 - 317. 